\title{
Cardiac tamponade: Rare presentation of acquired pericardial-esophageal fistula
}

\author{
Carmelo Mignosa, MD, FETCS, , Salvatore Agati, MD, ${ }^{\text {a }}$ Placido Gitto, MD, \\ Dario Salvo, MD, ${ }^{c}$ and Eugenio Santo Trimarchi, MD, ${ }^{a}$ Taormina, Italy
}

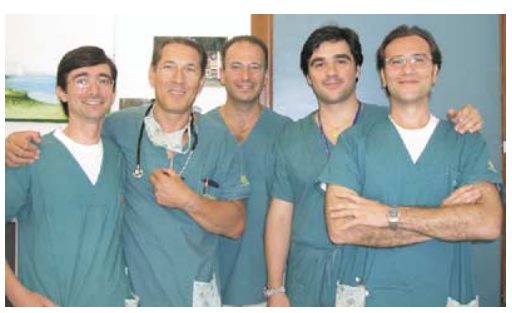

\section{Supplemental material is available online.}

$\mathrm{F}$ oreign body ingestion in the upper digestive tract is a common problem for children and adults. Estimated annual incidence in the United States is about 120 per million inhabitants, with approximately 1500 deaths each year. ${ }^{1}$ Foreign body ingestion can cause perforation of the thoracic esophagus, and it must be part of the differential diagnosis along with pleuritis, pericarditis, and pancreatitis. A prompt and aggressive nonoperative approach of esophageal perforations in children allows survival and conservation of the organ and its function in most cases and should remain the first therapeutic choice for young patients. We report the case of an infant with acquired pericardial-esophageal fistula complicated by low cardiac output syndrome.

\section{Clinical Summary}

A 15-month-old baby was transferred to our department on an emergency basis because of clinical signs of low cardiac output syndrome after abdominal surgery for foreign body removal. She had been admitted to the referring hospital for accidental foreign body ingestion (Christmas ornament) that had happened 20 days earlier. At her arrival, an esophagogram showed the presence of a metallic body positioned in the esophagus at its supracardiac portion (Figure 1). Endoscopic removal was attempted unsuccessfully. The patient underwent surgery, and the foreign body was removed through a gastronomy approach. No further procedures were done at the level of the lesion. Postoperative endoscopic examination showed the presence of an irregular, small, fibrin-coated necrotic ulcer. Forty-eight hours after the operation, enteral feeding was resumed. One day after the operation, clear signs of low cardiac output syndrome with oliguria were seen. Transthoracic echocardiography (TTE) showed pericardial effusion.

\footnotetext{
From the Pediatric Cardiac Surgery Unit, ${ }^{\mathrm{a}}$ Pediatric Cardiology Unit, ${ }^{\mathrm{b}}$ and Pediatric Intensive Care Unit, ${ }^{c}$ San Vincenzo Hospital, Taormina, Italy.

Received for publication June 13, 2005; accepted for publication July 7 , 2005.

Address for reprints: Salvatore Agati, MD, Pediatric Cardiac Surgery Unit, San Vincenzo Hospital, Contrada Sirina, 98039, Taormina (Messina), Italy (E-mail: sasha.agati@tiscali.it).

J Thorac Cardiovasc Surg 2005;130:1711-2

$0022-5223 / \$ 30.00$

Copyright $\odot 2005$ by The American Association for Thoracic Surgery

doi:10.1016/j.jtcvs.2005.07.015
}

On admission to our unit, the patient had fever and was irritable, tachycardiac, and tachypneic. She was peripherally cold and hypotensive, with narrow pulse pressure (systolic pressure $60 \mathrm{~mm} \mathrm{Hg}$ ) and pulsus paradoxus. On TTE, the right ventricle was seen to be compressed by high-echo contrast effusion (Figure E1), with severely impaired biventricular function and moderate mitral valve incompetence. Preoperative chest radiography showed an enormous dilatation of the mediastinum, with signs of pneumopericardium (Figure E2).

The patient underwent surgery on an emergency basis. Subxiphoid surgical pericardiocentesis was tried as a primary approach. Because of the impossibility removing effusions and cleaning pericardial space, a median sternotomy was performed (Figure 2). At opening, there was foul-smelling air emission, and the epicardial surface and the visceral pericardium were covered by a white film that rendered cardiac structures unrecognizable. Several specimens were obtained at different sites. The pericardium was accurately débrided, and a wide hole was found in the posterior mediastinum. It was closed with pledget-supported 4-0 Prolene suture (Ethicon, Inc, Somerville, NJ). The pericardium was left open, and the sternum was closed in the usual fashion over two chest drains operating on conventional underwater, high-pressure suction.

Postoperative TTE showed moderate mitral regurgitation and biventricular dysfunction with a pulmonary artery pressure of 50 $\mathrm{mm} \mathrm{Hg}$. The patient was maintained with inotropic support (epinephrine $0.1 \mu \mathrm{g} /[\mathrm{kg} \cdot \mathrm{min}]$, dobutamine $5 \mu \mathrm{g}[\mathrm{kg} \cdot \min ])$ for 72 hours. All the specimens showed positive Gram staining. On the 16th postoperative day, a contrast esophagogram excluded the presence of any communication between the esophagus and surrounding spaces. Enteral nutrition was then restarted to reach a normal regimen within 3 days. Progressive improvement of clinical conditions allowed us to discharge the baby 25 days after the operation. At 2-year follow-up, she was free of symptoms, and a TTE showed normal biventricular function with competent mitral valve.

\section{Discussion}

Cardiac tamponade caused by acquired pericardial-esophageal fistula is rare, and emergency pericardiocentesis and surgical revision remain life-threatening procedures. Outcomes have been correlated both with early diagnosis and with appropriate surgical management. Delayed diagnosis has been associated with high morbidity and mortality. Reported mortality for acute pericardial effusion in the neonatal population, resulting the lack of warning signs and sudden decompensation, has been estimated at $45 \%$ to $67 \% .^{2}$

Esophageal perforation requires prompt and vigorous treatment. In a large series of adult patients, ${ }^{3}$ it was clearly demon- 


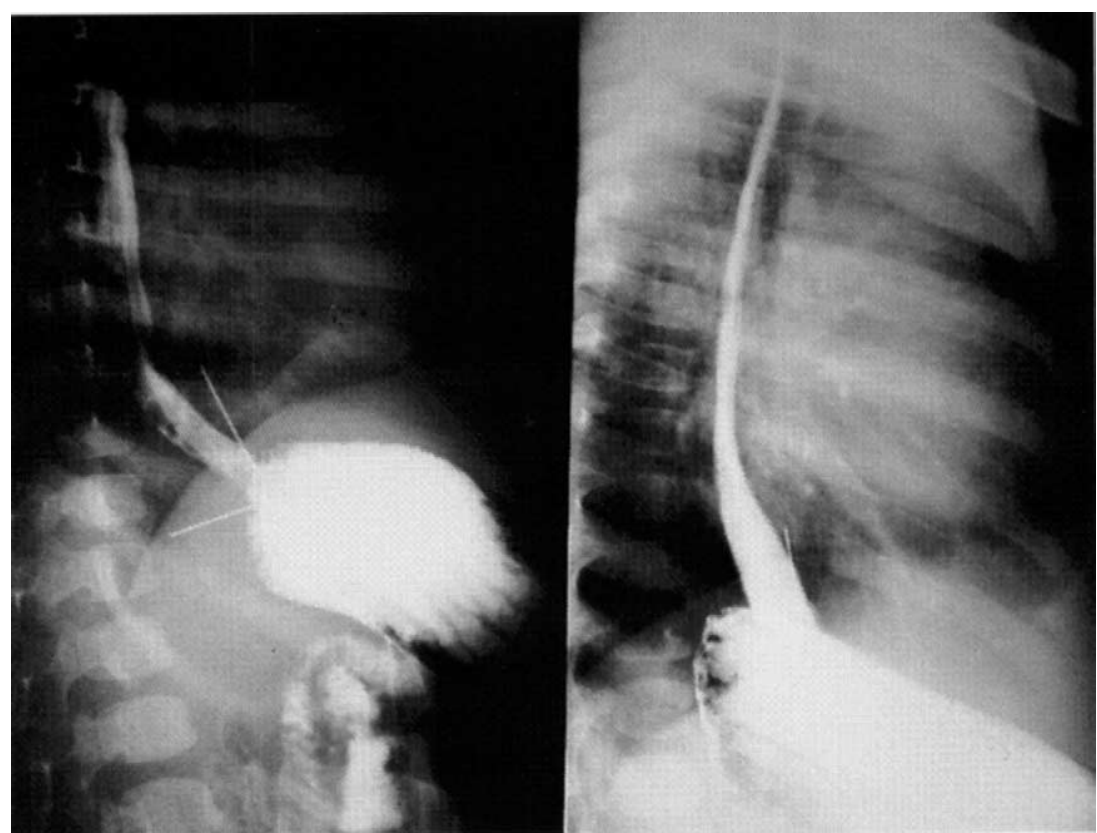

Figure 1. Esophagogram showing foreign body.

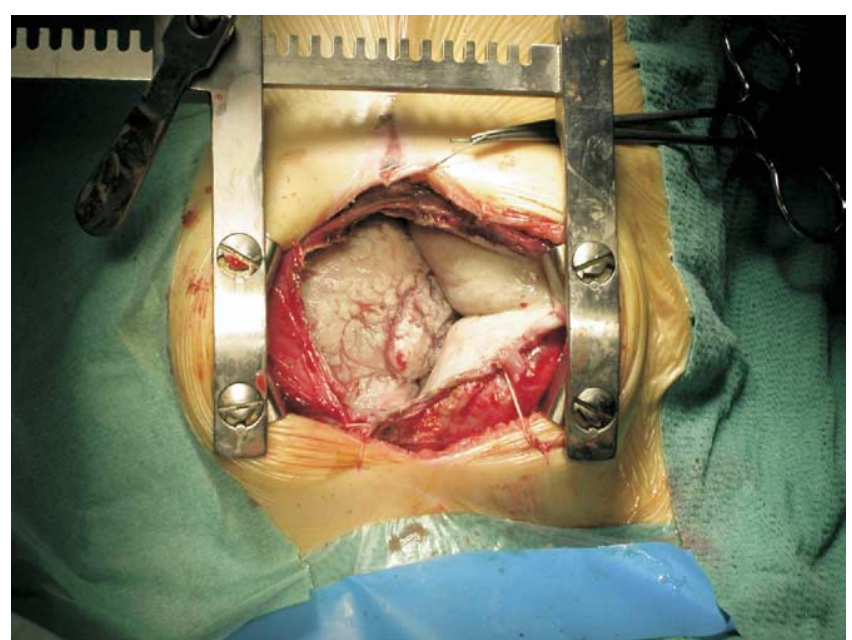

Figure 2. Intraoperative findings.

strated that esophagectomy is superior to primary repair alone in the presence of an esophageal lesion occurring more then 24 hours from presentation. Unlike the practice with adult patients, nonoperative treatment has been the usual approach for children. ${ }^{4,5}$ In our case, we treated the condition as acute mediastinitis because of acute worsening of the clinical status, fever, and rising inflammatory markers. Aggressive mediastinal débridement and wide mediastinal drainage with negative vacuum-assisted suction were performed. Antibiotics were given against both gram-positive and gram-negative bacteria and against both anaerobic and aerobic bacteria. Antifungal therapy was used as an adjunct both because of positive specimens and to prevent superinfections. The situation we faced as the result of incorrect postoperative management could have been lethal for this infant.

\section{References}

1. Chaudhary AM. Foreign bodies in the upper gastrointestinal tract. Kans Med. 1987;88:116-8.

2. Keeney SE, Richardson CJ. Extravascular extravasation of fluid as a complication of central venous lines in the neonate. J Perinatol. 1995; 15:284-8.

3. Asensio JA, Chawan S, Forno W. Penetrating esophageal injuries: multicenter study of the American Association for the Surgery of Trauma. J Trauma. 2001;50:289-96.

4. Rivas S, Martinez L, Hernandez F, Avila LF, Lassaletta L, Murcia J, et al. [Aggressive conservative treatment remains the best option for esophageal perforation in children.] Spanish. Cir Pediatr. 2004;17:3-7.

5. Kerschner JE, Beste DJ, Conley SF. Mediastinitis associated with foreign body erosion of the esophagus in children. Int J Pediatr Otorhinolaryngol. 2001;59:89-97. 


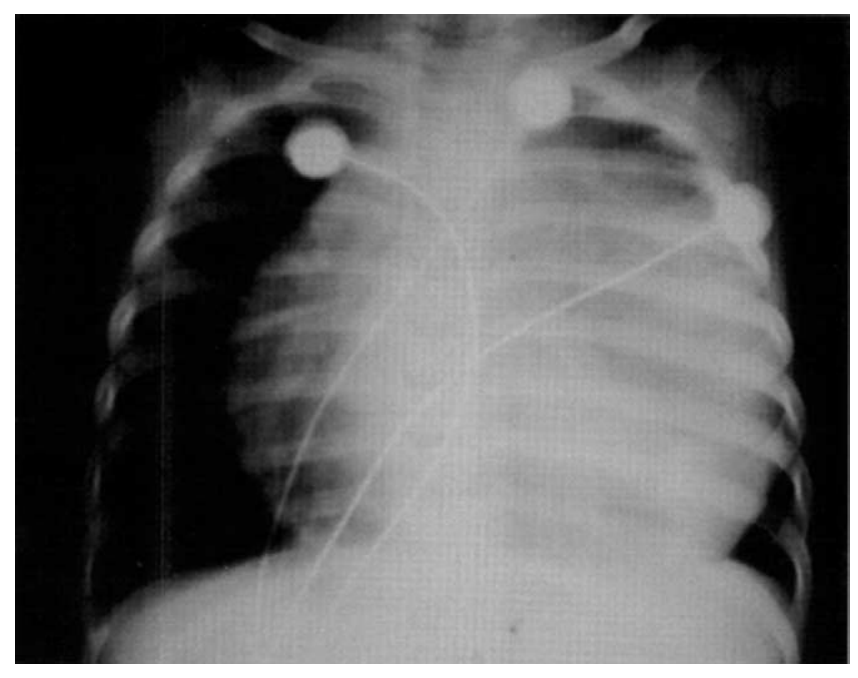

Figure E1. Preoperative chest radiograph.

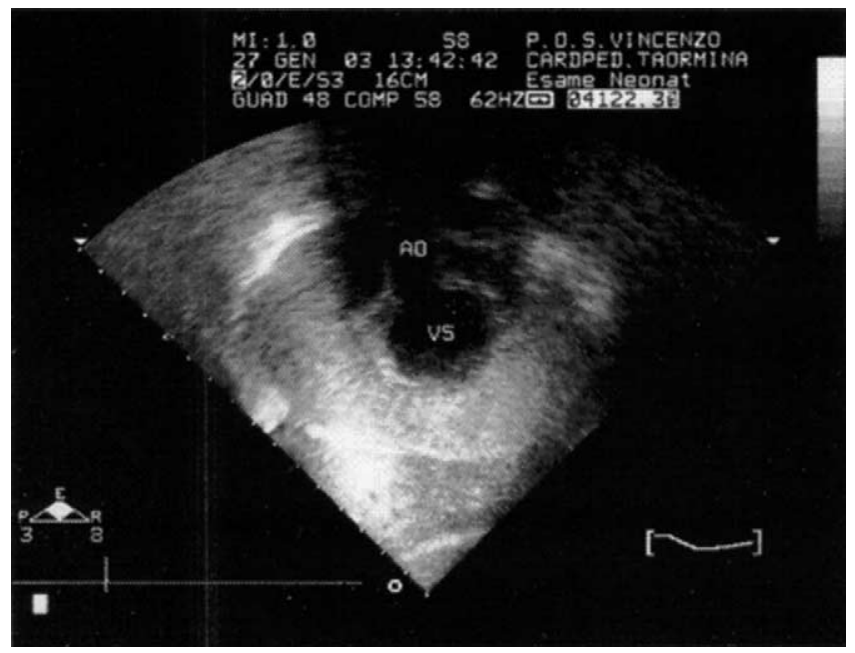

Figure E2. Preoperative TEE. 